\title{
The X-ray polarimetry window reopens
}

\author{
PolarLight is the first dedicated soft X-ray polarimeter in space since the 1970s and is attempting to reopen this \\ long-awaited window in astronomy, explain Principal Investigator Hua Feng and Ronaldo Bellazzini.
}

P olarLight ${ }^{1}$ is a CubeSat payload that utilizes a novel technique to detect $\mathrm{X}$-ray polarization in the standard band (a few keV) of X-ray astronomy. This is a window that has remained closed in astronomy for more than 40 years - mainly due to the lack of high-sensitivity techniques - with the previous experiment ${ }^{2}$ dating back to the 1970s. In 2001, a new technique for high-sensitivity X-ray polarimetry was demonstrated as possible in the laboratory ${ }^{3}$ and has been developed since then ${ }^{4}$. Future space missions based on this new technique, such as the Imaging X-ray Polarimetry Explorer (IXPE) ${ }^{5}$ and the enhanced X-ray Timing and Polarimetry (eXTP) ${ }^{6}$ mission, are planned.

In the new technique, dimethyl ether (DME) gas is sealed in a chamber with a beryllium entrance window. DME absorbs incident X-rays, generating photoelectrons that start to ionize DME molecules, ejecting further electrons. A high voltage is applied between the beryllium window, which acts as a cathode, and an anode. Driven by the electric field, electrons move toward the anode, following field lines that permeate micro-holes in a thin foil called the gas electron multiplier (GEM). Strong electric fields are formed in the micro-holes, which generate avalanches of electrons. These secondary electrons, with a high enough signal-to-noise ratio, are collected by an application-specific integrated circuit (ASIC) chip with hexagonal pixels (the anode). This assembly is called a gas pixel detector (GPD), shown in Fig. 1. A piece of capillary glass on top of the GPD is used as a collimator to shield most of the cosmic X-ray background. The sensitive area of the detector is defined by the ASIC, which has a geometry of $1.5 \mathrm{~cm}$ $\times 1.5 \mathrm{~cm}$, giving a collecting area of $1.6 \mathrm{~cm}^{2}$ when the open fraction of the collimator is taken into account. X-rays are admitted to the collimator from a field of view of 2.3' (full width at half maximum). Additionally, considering the absorption of the window and the stopping power of the gas, the effective area of the detector, which is a function of energy, has a peak of $0.18 \mathrm{~cm}^{2}$ at $2.5 \mathrm{keV}$. These numbers indicate that PolarLight is indeed a tiny instrument in the space.

For each X-ray event, a two-dimensional image of the charge deposit caused by the photoelectron projected on the detector plane is saved (also illustrated in Fig. 1). The
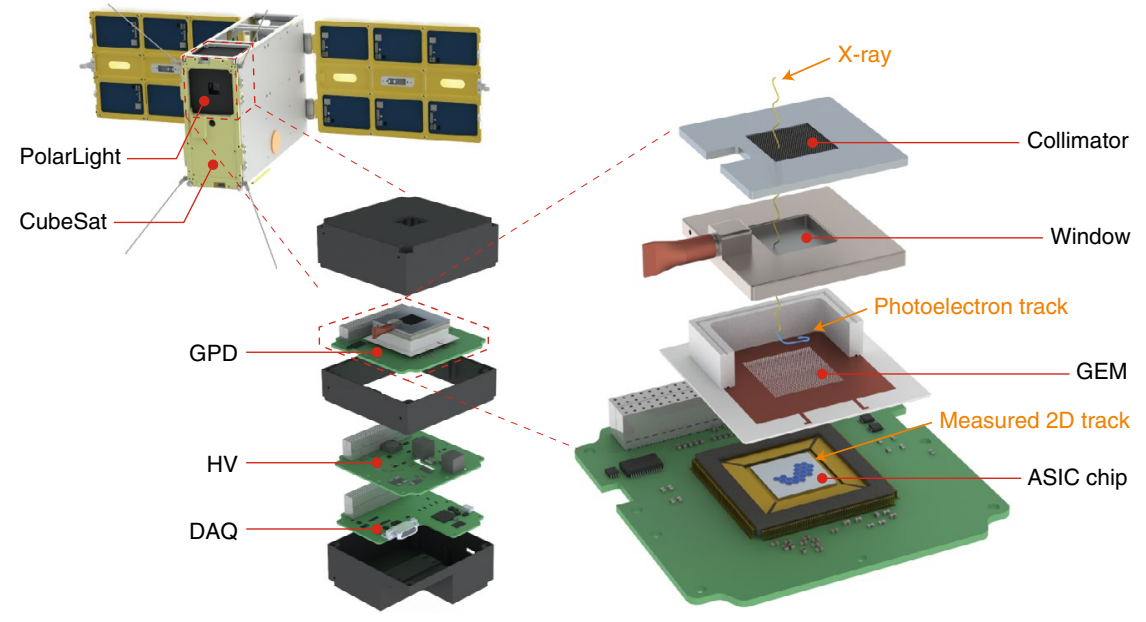

Fig. 1 | Schematic drawings of the CubeSat, the PolarLight payload and its internal GPD. The right-hand side illustrates how a photoelectron track is measured following the absorption of an X-ray.

shape of the image gives the motion of the photoelectron. Owing to its small mass, the photoelectron is easily scattered, resulting in non-straight tracks. In the energy range of interest, the photoelectron will lose a larger fraction of its energy at the end of the track than at the beginning. Thus, the lighter end of the track image indicates the interaction point, from which the initial direction of the photoelectron can be inferred. This initial direction - that is, the azimuthal angle of the ejected photoelectron - encodes the polarization of the incident X-ray and is the key parameter to be measured.

The GPD is mounted on a printed circuit board. There are two other boards in PolarLight, one for high-voltage (HV) generation and the other for data acquisition (DAQ) and communication. The three boards stack vertically and are encircled by an aluminium case, which has a size of about $10 \mathrm{~cm} \times 10 \mathrm{~cm} \times 10 \mathrm{~cm}$ and occupies a standard unit $(1 \mathrm{U})$ of a CubeSat.

PolarLight is on-board a $6 \mathrm{U}$ CubeSat named Tongchuan-1, manufactured by Spacety, and launched into a Sun-synchronous orbit on 29 October 2018. PolarLight started regular science observations in March 2019 after a commissioning phase, and to date is still in good health. To avoid HV discharges, PolarLight is powered off when passing through regions with high charged-particle flux. Every day, a student is responsible for generating and uploading the commands needed for satellite and payload control for the next day. Thus PolarLight also serves as a good platform for student training.

PolarLight shares the CubeSat with two other payloads in order to minimize cost. The baseline objective of PolarLight is to have a direct demonstration of the new GPD technique in space and a better understanding of the in-orbit background. As X-ray polarimetry is a relatively unexplored window, scientific returns are also expected. Despite its small collecting area, a well-defined science plan could probe parameter spaces that are practically uncovered by large observatories. For example, staring at a single or a few objects for months to years may sound extravagant for large observatories, but could result in interesting science (see ref. ${ }^{7}$ for an example).

Hua Feng (D) ${ }^{1 凶}$ and Ronaldo Bellazzini ${ }^{2}$ ${ }^{1}$ Department of Astronomy, Tsinghua University, Beijing, China. ${ }^{2}$ Istituto Nazionale di Fisica Nucleare (INFN-Pisa), Pisa, Italy.

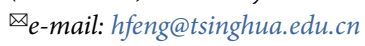

Published online: 14 May 2020 https://doi.org/10.1038/s41550-020-1103-6

\footnotetext{
References

1. Feng, H. et al. Exp. Astron. 47, 225-243 (2019).

2. Weisskopf, M. C. et al. Astrophys. J. 208, L125-L128 (1976).

3. Costa, E. et al. Nature 411, 662-665 (2001).

4. Bellazzini, R. et al. Nucl. Instrum. Meth. A 720, 173-177 (2013).

5. Weisskopf, M. C. et al. Proc. SPIE 9905, 990517 (2016).

6. Zhang, S. et al. Sci. China Phys. Mech. Astron. 62, 29502 (2019)

7. Feng, H. et al. Nat. Astron. https://doi.org/10.1038/s41550-020 1088-1 (2020).
} 